This document is confidential and is proprietary to the American Chemical Society and its authors. Do not copy or disclose without written permission. If you have received this item in error, notify the sender and delete all copies.

\title{
Computational Screening of Structure Directing Agents for the Synthesis of Pure Silica ITE Zeolite
}

\begin{tabular}{|r|l|}
\hline Journal: & The Journal of Physical Chemistry Letters \\
\hline Manuscript ID & jz-2020-017343.R2 \\
\hline Manuscript Type: & Letter \\
\hline Date Submitted by the \\
Author: & n/a \\
\hline Complete List of Authors: & $\begin{array}{l}\text { León, Santiago; Instituto de Tecnologia Quimica } \\
\text { Sastre, German; Instituto de Tecnologia Quimica, }\end{array}$ \\
\hline
\end{tabular}

\section{SCHOLARONE \\ Manuscripts}




\title{
Computational Screening of Structure Directing
}

\section{Agents for the Synthesis of Pure Silica ITE Zeolite}

\author{
Santiago León, German Sastre* \\ Instituto de Tecnología Química (UPV-CSIC), Universidad Politécnica de Valencia, Av. Naranjos \\ s/n, 46022 Valencia, Spain \\ E-mail: gsastre@,itq.upv.es
}




\begin{abstract}
Shape' was the first criteria claimed to explain specificity between organic structure directing agents (OSDAs) and zeolite micropores. With the advent of computational chemistry methods applied to study the effectiveness of SDA-zeolite combinations, 'energy' (mainly van der Waals) became the most commonly invoked concept to explain zeolite phase selectivity. The lower the energy the better the SDA. In this study we rescue the concept of 'shape' and we combine it with the concept of 'energy' within the frame of a SDA screening approach to identify new SDAs for the synthesis of cage-based ITE zeolite. Once we identify an appropriate 'shape' fingerprint, filtering through the SDA database can be made fast and accurately. With the 'shape' selection, an automated Monte Carlo software allows to assess suitability using the force-field calculated zeo-SDA 'energy'. The computational approach can be promptly applied to other cage-based zeolites.
\end{abstract}

\title{
TOC GRAPHICS
}

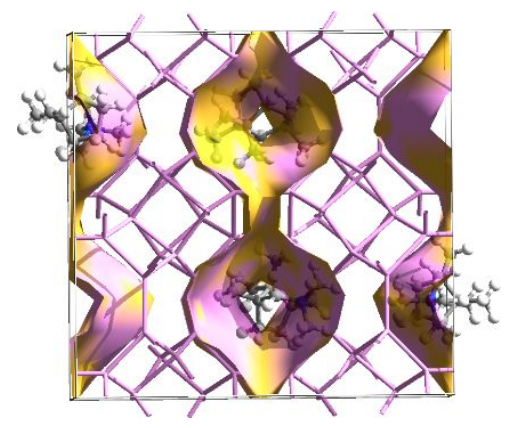

$5 \mathrm{~cm} \times 5 \mathrm{~cm}$ graphic

KEYWORDS: database screening, zeolites, Monte Carlo, force field 
Zeolites are a large and growing family of crystalline microporous alumino-silicates with important applications as selective adsorbents and catalysts. Obtaining new and modify existing zeolites is crucial in order to improve these applications, and this can be achieved by finding new organic molecules that can act as templates (structure directing agents, SDA) for their synthesis $[1,2,3]$

Many variables influence the synthesis process of a zeolitic material, making difficult to establish a relationship between synthetic variables and the particular zeolite structures formed [4]. Although relevant synthesis parameters are known, such as crystallization time, temperature, mineralization agent, solvent, $\mathrm{pH}$, gel composition, structure-directing agent (SDA), concentration, a rational selection of the parameters necessary to drive the synthesis towards a specific zeolite is yet not possible, in spite of six decades [5] of intensive work.

Among the early attempts in trying to control how the synthesis variables determine the zeolite formed, the pioneer work of Zones [6] and Davis [7], among others [8], focused on the important role of the organic SDA (OSDA), particularly at high Si/Al ratio. This role is not only important but can also be rationalized into the intuitive concept of host-guest geometric (size and shape) match [9], that we will use in this study. This concept is entirely due to the hypothesis that the dominant host-guest interactions are short range, i.e. van der Waals interactions. A perfect match is, however, rarely found and in fact not needed, as demonstrated by the frequent rotational capability of OSDAs inside the zeolite micropores. The host-guest match concept was soon found to open an active topic in computational chemistry [10]. Within a few years it was possible to apply force field based methods not only to particular zeolita-OSDA systems [11], but also to series of zeolite- $\{$ OSDA $\}$ i or \{zeolite $\}$ i-OSDA $[12,13,14]$. This has been recently expanded with the advent of artificial intelligence algorithms, making possible the automated analysis of big data, allowing to select or design new SDAs using large databases or molecular building rules [15,16,17,18,19].

In this study we will use the latter approach, following our previously established methodology $[15,16]$ and including some novelties, in order to design new SDAs for ITE (ITQ-3 [20]) zeolite, 
with potential applications in separation of light hydrocarbons [21]. We developed two additional tools to improve our existing computational methodology: a) the selection of a large number of OSDAs from SciFinder database, and b) a new algorithm named 'shoebox' to search OSDA molecules that fit in a zeolite cavity.

Instead of choosing among conventional databases of organic molecules employed in a previous publication [16], we have tried to enlarge our previous database of 241 OSDAs [15]. For this task, we have taken quaternary ammonium monocations from SciFinder [22], allowing to expand the database up to 651 molecules. Notation of the SDAs appears with SciFinder codes that contain the corresponding quaternary ammonium cation, regardless the counteranion, that has not been considered. Additionally, we have selected from the literature 12 SDAs reported to drive the synthesis towards ITE zeolite (ITQ-3 and SSZ-36, a faulted intermediate between the RTH and ITE) $[12,14,20]$, whose CAS codes are indicated in Table 1 and Figure 1.

The shoebox algorithm, inspired on previous calculations of molecular size [9], finds the two atoms of maximum intramolecular distance and makes a rotation to define a new x-axis along those two atoms. In the new coordinate system, the maximum and minimum coordinates over the three axis define a box (xmax-xmin, ymax-ymin, zmax-zmin) that we call shoebox and whose values, ordered from largest to shortest (D1, D2, D3, respectively) are our definition of SDA size. More details are given as Supporting Information. The resulting dimensions of the SDAs employed in the synthesis of ITE are included in Table 1. Using the value of the largest sphere that can be included [23] (MxSp) in ite cavity $(8.3 \AA)$, it is useful to calculate $\mathrm{D} 1 / \mathrm{MxSp}$ ( $\varphi$, Table 1$)$, whose value should be not far from unity assuming host-guest match. From the spheric-like symmetry of ite cavity, D3/D1 and D2/D1 would ideally be close to unity, but in reality (see Table 1) they tend to be larger than 0.6.

From the above analysis, we consider that suitable SDAs for ITE zeolite should obey the following three conditions:

$$
0.75<\mathrm{D} 1 / \mathrm{MxSp}<1.00 ; \quad \mathrm{D} 2 / \mathrm{D} 1>0.6 ; \quad \mathrm{D} 3 / \mathrm{D} 1>0.6
$$


Table 1. SDAs employed in the literature for the synthesis of ITE (ITQ-3, SSZ-36). Size-related parameters D1, D2, D3 $(\AA), \varphi=\mathrm{D} 1 / \mathrm{MxSp}(\mathrm{MxSp}$ : largest sphere that can be included in ITE). Van der Waals ITE-SDA interactions $\left(\mathrm{E}_{\mathrm{vdW}}, \mathrm{kJ} / \mathrm{mol}\right)$ calculated per each SDA molecule. The values in $\mathrm{kJ} / \mathrm{Si}$, at full loading (four molecules per unit cell), can be obtained by applying the factor 4/64.

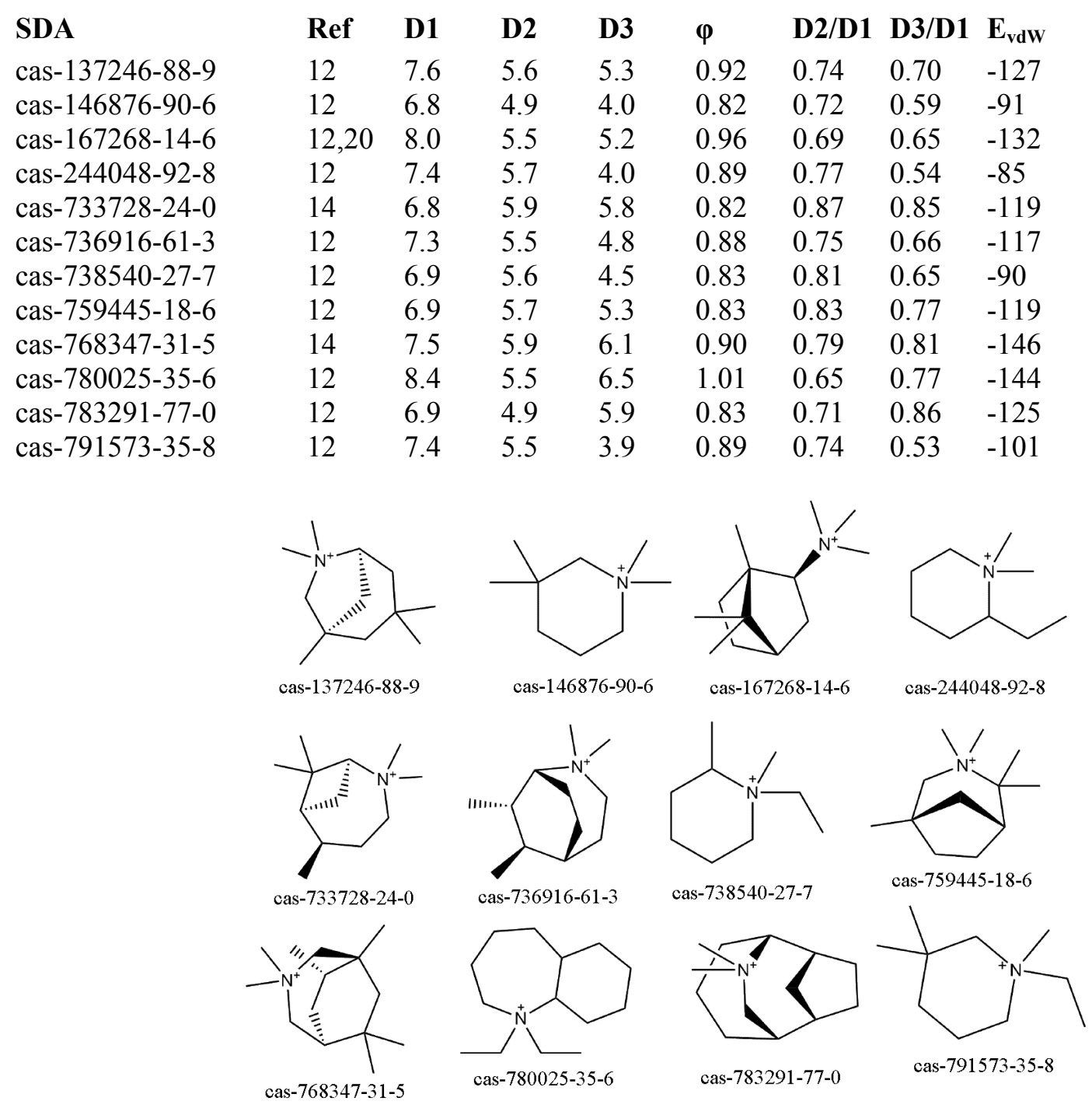

Figure 1. Organic SDAs employed in the literature for the synthesis of ITE (see Table 1).

In order to confirm whether van der Waals interaction energy between SDA and ITE structure is a relevant parameter to discriminate between suitable and unsuitable SDAs, we calculated those energies using a Monte Carlo + Lattice Energy Minimization approach using the software zeoTsda [15] (more details are given as Supporting Information). The software includes both: low loading 
(one OSDA molecule per unit cell) and full loading, which for ITE corresponds to 4 molecules per unit cell (64 SiO2). The results $\left(\mathrm{E}_{\mathrm{vdW}}\right)$ are shown in Table 1, and indicate an energy interval of $[-85,-146] \mathrm{kJ} / \mathrm{mol}$ for the stabilization energy of one OSDA molecule per ite cavity. Hence, we expect that alternative suitable SDAs for ITE zeolite should have interaction energies within the same interval.

Next, we run the shoebox algorithm through the database (651 SDAs) and we select those meeting the three geometrical conditions in equations 1-3. The SDAs selected are shown in Figure 2, with their calculated geometric data, including size and van der Waals stabilisation energy. For SDAs containing chiral carbons, only the enantiomer shown in Table 2 has been considered.

Table 2. SDAs from database meeting the three conditions of suitability for ITE zeolite: $0.75<$ $\mathrm{D} 1 / \mathrm{MxSp}<1.00, \mathrm{D} 2 / \mathrm{D} 1>0.6, \mathrm{D} 3 / \mathrm{D} 1>0.6$. Van der Waals ITE-SDA interactions $\left(\mathrm{E}_{\mathrm{vdW}}\right)$ calculated per each SDA molecule. The values in $\mathrm{kJ} / \mathrm{Si}$, at full loading (four molecules per unit cell), can be obtained by applying the factor $4 / 64$.

$\begin{array}{llllllll}\text { SDA } & \text { D1 } & \text { D2 } & \mathbf{D 3} & \boldsymbol{\varphi} & \mathbf{D 2} / \mathbf{D 1} & \mathbf{D 3} / \mathbf{D} 1 & \mathbf{E}_{\mathbf{v d W}} \\ \text { cas-129024-58-4 } & 7.5 & 4.7 & 6.0 & 0.90 & 0.63 & 0.80 & -103 \\ \text { cas-146876-82-6 } & 7.5 & 5.6 & 5.9 & 0.90 & 0.75 & 0.79 & -135 \\ \text { cas-166746-03-8 } & 7.3 & 6.4 & 6.0 & 0.88 & 0.88 & 0.82 & -120 \\ \text { cas-1677677-86-9 } & 7.0 & 5.8 & 4.4 & 0.84 & 0.83 & 0.63 & -102 \\ \text { cas-1918193-04-0 } & 7.1 & 5.9 & 5.4 & 0.86 & 0.83 & 0.76 & -121 \\ \text { cas-1918193-05-1 } & 7.3 & 5.3 & 5.1 & 0.88 & 0.73 & 0.70 & -89 \\ \text { cas-2242581-09-3 } & 7.5 & 5.8 & 5.4 & 0.90 & 0.77 & 0.72 & -128 \\ \text { cas-244048-95-1 } & 6.9 & 5.9 & 5.8 & 0.83 & 0.86 & 0.84 & -87 \\ \text { cas-25728-39-6 } & 7.3 & 4.2 & 4.2 & 0.88 & 0.58 & 0.58 & -87 \\ \text { cas-38018-80-3 } & 6.9 & 6.1 & 5.6 & 0.83 & 0.88 & 0.81 & -128 \\ \text { cas-400825-31-2 } & 7.4 & 6.2 & 5.5 & 0.89 & 0.84 & 0.74 & -120 \\ \text { cas-51780-27-9 } & 7.4 & 5.7 & 6.6 & 0.89 & 0.77 & 0.89 & -119 \\ \text { cas-918306-90-8 } & 7.9 & 5.9 & 5.9 & 0.95 & 0.75 & 0.75 & -139 \\ \text { cas-941-14-0 } & 7.2 & 4.9 & 5.2 & 0.87 & 0.68 & 0.72 & -118 \\ \text { cas-94267-29-5 } & 6.8 & 5.9 & 4.4 & 0.82 & 0.87 & 0.65 & -103 \\ \text { cas-96900-94-6 } & 8.0 & 6.2 & 5.8 & 0.96 & 0.78 & 0.73 & -139\end{array}$




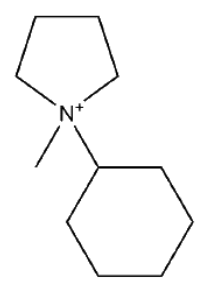

cas-129024-58-4

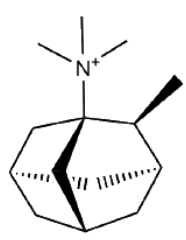

cas-146876-82-6

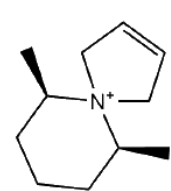

cas-1918193-04-0 cas-1918193-05-1

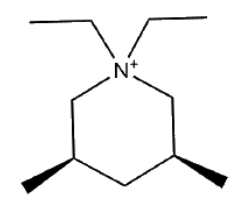

cas-166746-03-8

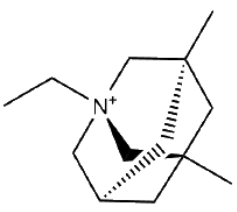

cas-2242581-09-3

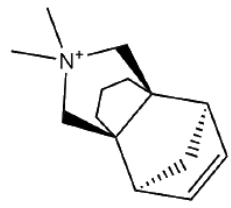

cas-400825-31-2

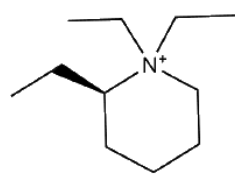

cas $-51780-27-9$

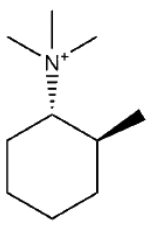

cas-94267-29-5

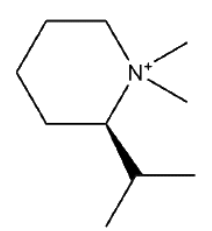

cas-1677677-86-9

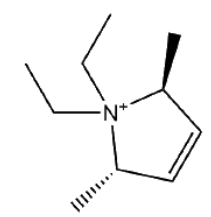

cas-244048-95-1

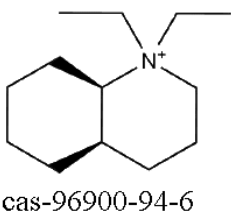

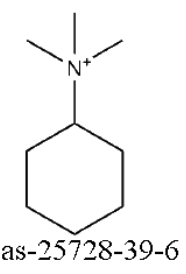

cas-38018-80-3

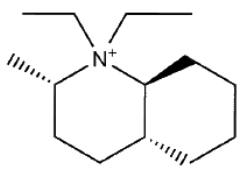

cas-918306-90-8

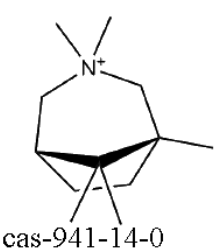

Figure 2. New candidate organic SDAs for the synthesis of ITE (see Table 2).

A comparison between Figures 1 and 2 allows to identify some similarities. Piperidinium derivatives such as cas-146876-90-6, cas-244048-92-8, cas-738540-27-7 and cas-791573-35-8 in Figure 1 can also be found in Figure 2 (cas-166746-03-8, cas-1677677-86-9, cas-1918193-04-0 and cas-51780-27-9). An azoniabicyclo-octane derivative (such as cas-137246-88-9, cas-733728-24-0 and cas-759445-18-6, Figure 1), is found in Figure 2 (cas-941-14-0). New chemical diversity appears in Figure 2 with: a pyrrolium derivative (cas-244048-95-1), pyrrolidinium derivative (cas129024-58-4), adamantane derivatives (cas-146876-82-6 and cas-2242581-09-3), 5azoniaspiro[4.5]decane derivative (cas-1918193-05-1), quinolinium-decahydro (cas-96900-94-6 and cas-918306-90-8) and cyclohexanaminium derivative (cas-94267-29-5), among others.

The SDA-ITE van der Waals interaction energies in Table 1 are in the interval $[-85,-146] \mathrm{kJ} / \mathrm{mol}$, similar to that in Table $2([-87,-139] \mathrm{kJ} / \mathrm{mol})$, indicating that the computational methodology is 
successful in finding candidate SDAs that stabilize ITE structure. Figure 3 (top) shows the final configuration of one of the candidate SDAs in Table 2, cas-941-14-0, with a strong van der Waals stabilisation $(-118 \mathrm{~kJ} / \mathrm{mol})$ and a largest dimension $(7.2 \AA)$ fitting tightly in ite cavity $(8.3 \AA)$. The fit of cas-941-14-0 in ite cavity, and, for comparison, that of cas-167268-14-6, which is the original SDA of ITE [20], are also shown (Figure 3, bottom).
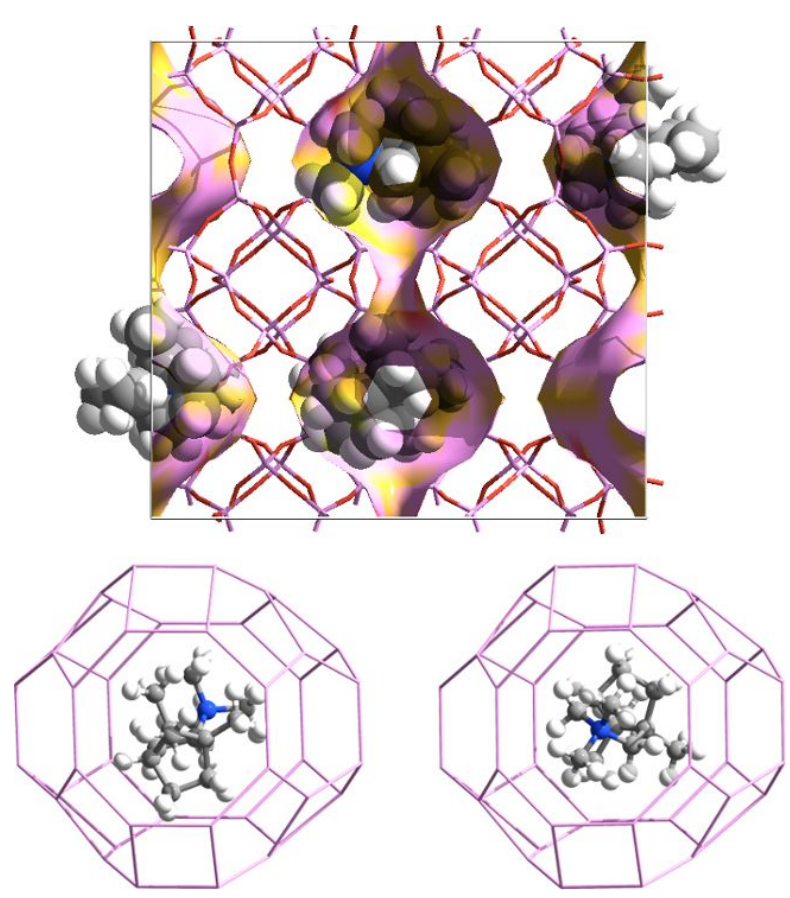

Figure 3. Top: SDA cas-941-14-0 in ITE. Bottom: Comparison of optimized conformations of cas167268-14-6 (left) and cas-941-14-0 (right) in ite cavity.

Figure 4 allows to rationalize the results in terms of size of the SDA and van der Waals stabilisation energy. An appropriate size descriptor has been defined for each SDA, $(\mathrm{D} 1+\mathrm{D} 2+\mathrm{D} 3) \times \mathrm{N}$, where $\mathrm{N}$ is the number of atoms, that shows a correlation with the van der Waals stabilization energy in ITE structure. Experimental SDAs of Table 1 (circles) and computationally found candidate SDAs of Table 2 (squares) fit equally well in this correlation, indicating that the computational methodology is giving results comparable to those of the experiments. 
The present computational methodology opens the way to suggest candidate SDAs for ITE just by calculating size descriptors, without the need of performing the more computationally demanding calculation of stabilization energy. The initial strategy of selecting three conditions regarding the SDA dimensions (D1,D2,D3) leads to a set of candidate SDAs (Table 2), all of which are included in the energy span expected for the synthesis of ITE (Figure 4). It has been possible to approximately rank the SDA suitability by defining a size descriptor. This simple strategy can be applied to any zeolites containing cavities and will contribute to suggest new SDAs for their synthesis.

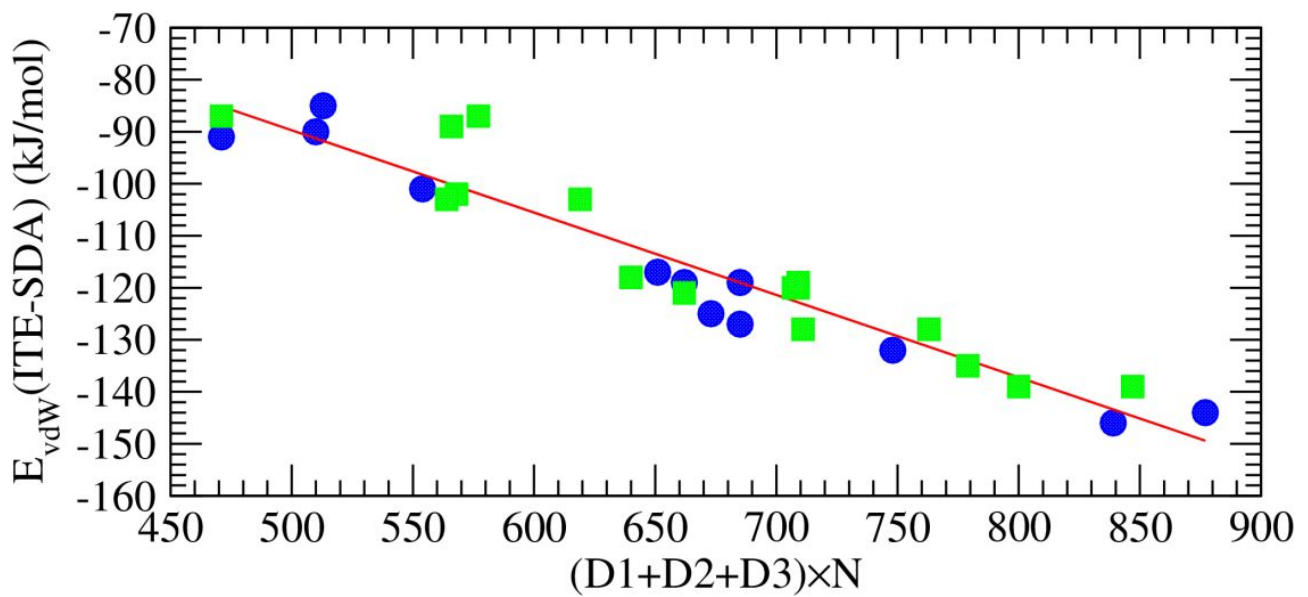

Figure 4. Correlation between SDA size parameter and van der Waals stabilization energy in ITE. Circles: SDAs in Table 1. Squares: SDAs in Table 2. Linear fit: $E_{\mathrm{vdW}}=-10.506$ $-0.1584 \times(\mathrm{D} 1+\mathrm{D} 2+\mathrm{D} 3) \times \mathrm{N}$. 


\title{
ASSOCIATED CONTENT
}

Supporting Information.

The Supporting Information is available free of charge at https://pubs.acs.org/doi/10.1021/acs.jpclett.xxxxxxx.

Computational details, additional data for Figure 4, comparison between ITE and RTH zeolites, figures of the optimized geometries of SDAs in ite cavity. The following files are free of charge: zeo_SDA_CIFs.zip (file type: ZIP), CIF files containing zeolite (ITE and RTH unit cells containing 4 optimized molecules of each SDA in Tables 1 and 2).

\author{
AUTHOR INFORMATION \\ Corresponding Author \\ *E-mail: gsastre@itq.upv.es Phone: +34 963879445.

\section{ORCID} \\ G. Sastre: 0000-0003-0496-6331
}

Notes

The authors declare no competing financial interest.

\section{ACKNOWLEDGEMENTS}

We thank MICINN of Spain for funding through projects RTI2018-101784-B-I00, RTI2018101033-B-I00, and SEV-2016-0683. S.L. thanks MICINN for a predoctoral grant BES-2017081245 corresponding to project SEV-2016-0683-17-2. Prof. A. Corma is acknowledged for collaboration from SEV-2016-0683 project. The authors thank ASIC-UPV for the use of their computational facilities. 


\section{References}

1 Davis, M. E.; Zeolites from a Materials Chemistry Perspective; Chem. Mater. 2014, 26, 239-245.

2 Li, Y.; Corma, A.; Yu, J.; Synthesis of new zeolite structures; Chem. Soc. Rev. 2015, 44, 71127127.

3 Čejka, J.; Millini, R.; Opanasenko, M.; Serrano, D. P.; Roth, W. J.; Advances and Challenges in Zeolite Synthesis and Catalysis; Catal. Today 2020, 345, 2-13.

4 Rimer, J. D.; Rational Design of Zeolite Catalysts; Nat. Catal. 2018, 1, 488-489.

5 Barrer, R. M.; Stabilization of lattices by sorbed and included molecules; J. Phys. Chem. Solids 1960, 16, 84-89.

6 Zones, S. I.; Synthesis of pentasil zeolites from sodium silicate solutions in the presence of quaternary imidazole compounds; Zeolites 1989, 9, 458-467.

7 Davis, M. E.; New Vistas in Zeolite and Molecular Sieve Catalysis; Acc. Chem. Res. 1993, 26, 111-115.

8 Gies, H.; Marler, B.; The structure-controlling role of organic templates for the synthesis of porosils in the system $\mathrm{SiO}_{2} /$ template/ $\mathrm{H}_{2} \mathrm{O}$; Zeolites 1992, 12, 42-49.

9 Boyett, R. E.; Stevens, A. P.; Ford, M. G.; Cox, P. A.; A quantitative shape analysis of organic templates employed in zeolite synthesis; Zeolites 1996, 17, 508-512.

10 Bell, R. G.; Lewis, D. W.; Voigt, P.; Freeman, C. M.; Thomas, J. M.; Catlow, C. R. A.; Computer modelling of sorbates and templates in microporous materials; Stud. Surf. Sci. Catal. 1994, 84, 2075-2082. 
11 Cox, P. A.; Casci, J. L.; Stevens, A. P.; Molecular modelling of templated zeolite synthesis; Faraday Discuss. 1997, 106, 473-487.

12 Wagner, P.; Nakagawa, Y.; Lee, G. S.; Davis, M. E.; Elomari, S.; Medrud, R. C.; Zones, S. I.; Guest/host relationships in the synthesis of the novel cage-based zeolites SSZ-35, SSZ-36, and SSZ-39; J. Am. Chem. Soc. 2000, 122, 263-273.

13 Shi, C.; Li, L.; Yang, L.; Li, Y.; Molecular simulations of host-guest interactions between zeolite framework STW and its organic structure-directing agents; Chinese Chem. Lett. 2020, in press; DOI: $\underline{10.1016 / \text { j.cclet.2020.01.016 }}$

14 Burton, A. W.; Lee, G. S.; Zones, S. I.; Phase selectivity in the syntheses of cage- based zeolite structures: An investigation of thermodynamic interactions between zeolite hosts and structure directing agents by molecular modeling; Microporous Mesoporous Mater. 2006, 90, $129-144$.

15 Gálvez-Llompart, M.; Cantín, A.; Rey, F.; Sastre, G.; Computational Screening of Structure Directing Agents for the Synthesis of Zeolites. A Simplified Model; Z. Kristallogr.- Cryst. Mater. 2019, 234, 451-460.

16 Gálvez-Llompart, M.; Gálvez, J.; Rey, F.; Sastre, G.; Identification of New Templates for the Synthesis of BEA, BEC and ISV Zeolites Using Molecular Topology and Monte Carlo Techniques; J. Chem. Inf. Model. 2020, 60, 2819-2829.

17 Schmidt, J. E.; Deem, M. W.; Davis, M. E.; Synthesis of a Specified, Silica Molecular Sieve by Using Computationally Predicted Organic Structure-Directing Agents; Angew. Chem. Int. Ed. 2014, 53, $8372-8374$.

18 Daeyaert, F.; Deem, M. W.; Design of Organic Structure Directing Agents for Polymorph A Zeolite Beta; J. Mater. Chem. A 2019, 7, 9854-9866. 
19 Daeyaert, F.; Ye, F.; Deem, M. W.; Machine-learning Approach to the Design of OSDAs for Zeolite Beta; Proc. Natl. Acad. Sci. U. S. A. 2019, 116, 3413-3418.

20 Camblor, M. A.; Corma, A.; Lightfoot, P.; Villaescusa, L. A.; Wright, P. A.; Synthesis and Structure of ITQ-3, the First Pure Silica Polymorph with a Two-Dimensional System of Straight Eight-Ring Channels; Angew. Chem. Int. Ed. Engl. 1997, 36, 2659-2661.

21 Olson , D. H.; Camblor, M. A.; Villaescusa, L. A.; Kuehl, G. H.; Light hydrocarbon sorption properties of pure silica Si-CHA and ITQ-3 and high silica ZSM-58; Microporous Mesoporous Mater. 2004, 67, 27-33.

22 SciFinder; CAS, P.O. Box 3012, Columbus, Ohio 43210 U.S.A. Copyright (C) 2020. American Chemical Society. https://www.cas.org/products/scifinder

23 Foster, M. D.; Rivin I.; Treacy, M. M. J.; Delgado Friedrichs, O.; A geometric solution to the largest-free-sphere problem in zeolite frameworks ; Microporous Mesoporous Mater. 2006, 90, 32-38. 